\title{
Emerging Trends in Coordination Polymers and Metal-Organic Frameworks: Perspectives, Synthesis, Properties and Applications
}

\author{
Nworie Felix Sunday* \\ Department of Industrial Chemistry, Ebonyi State University, Nigeria
}

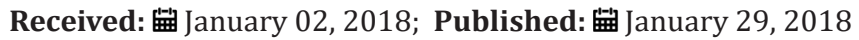

*Corresponding author: Nworie Felix Sunday, Department of Industrial Chemistry, Ebonyi State University, Nigeria, Tel: +2348034813342;

Email: nworie.felix@gmail.com

\begin{abstract}
Coordination polymers including metal-organic frameworks, clathrates and cyanometallates have recently gained significance as an important class of solid state materials. In this critical review, the diversity, classification, synthesis, structure, properties, applications, geometrical and chemical factors that influence their formation have been carefully presented. The synthesis of these new classes of compounds from molecular building blocks and demonstration of their high surface area, adsorptive and trapping potentials of molecules, selectivity in separation and uptake of molecules, tunable and modifiable molecular structure, and photoluminescence, magnetic and antimicrobial responses holds the potential for innumerable applications based on these tailored properties.
\end{abstract}

Keywords: Coordination polymers; Properties; Applications; Metal organic frameworks

Abbreviations: MOFs: Metals-Organic Frameworks; CPs: Coordination Polymers; SBUs: Secondary Building Units; PCPs: Porous Coordination Polymers; HSAB: Hard Soft Acid Base; KTP: Potassium Titanyl Phosphate; CT: Computed Tomography

\section{Introduction}

The development of polymer materials with coordination complexes as repeating units has increased in recent years .In the past decade, there have been rapid growth in coordination polymers and this defines one of the most exciting fields in solid state chemistry. The surprising variety of structures, compositions and properties of these polymers as well as their easy modification and synthesis has attracted many researchers [1]. As a consequence of the various synthetic procedures followed with modifications of the structures of these polymers, solid state chemistry has moved in several different directions depending on the particular property being investigated. Coordination polymers have very many unique properties that its applications can never be over emphasized. Their relevance in many fields like organic and inorganic chemistry, biochemistry, material science, electrochemistry and pharmacology with very many potential applications has led to extensive study of these polymers. Coordination polymer is a coordination compound or an inorganic or organ metallic polymer structure having metal cation centers connected by organic ligands with repeating coordination entities extending in one, two or three dimensions [2].

Coordination polymers have the subclass coordination networks which are coordination compounds extending through repeating coordination entities in one dimension but with crosslinks between two or more individual chains, loops, or spirolinks or a coordination compound extending through repeating coordination entities in two or three dimensions or metals-organic frameworks (MOFs) a coordination network that have organic ligands containing potential voids. Various authors have defined the concept based on their perceptive but all are the same or nearly related. Rowsell \& Yaghi [3] noted that coordination polymers (CPs) are solid species produced by metal clusters ligated to polydentate organic complexones. By this definition, enormous variety of species containing organic molecules and metals defined by characteristic properties such as porosity, non-porosity, crystallinity and amorphosity with emphasis on metal organic frameworks (MOFS) 
as special group of coordination polymers. The terms coordination polymers and metal organic frameworks have been currently used interchangeably. Cheetham et al. [4] noted that coordination polymers, or metal organic frameworks (MOFs) defines extended network arrays of metal clusters joined to polyfunctionally defined organic specie.

Therefore, coordination polymers (CPs) within the last decade emerged as hybrid materials made up of organic bridging ligands coordinative bonded to metal ions or metal ion clusters which when crystalline and three-dimensional are often regarded as metal-organic frameworks (MOFs). Metal organic frameworks (MOFS) according to O' Keeffe [5] used to be interchangeably used as coordination polymers but it specifically apply to materials with frameworks built by linking polyatomic clusters (secondary building units or SBUs) formed holistically by strong covalent bonds unlike coordination polymers which have some interplay of weaker bonds and lower stability. Long \& Yaghi [6] observed that inorganic joints are linked with organic units regarded as struts through strong interaction to yield non-porous or porous metal organic framework wrapped in architecturally extended network. Thus, the term "coordination polymers" refers to molecular crystal assembly or systems in which metal atoms multitypically coordinates to organic components. The metal organic framework currently discussed as reticular chemistry include components linked by strong bonds as indicated in metal carboxylates and is differentiated from coordination polymers.

\section{Classification of coordination polymers}

Coordination polymers can be classified in different ways according to their structure and composition into porous coordination polymers and non-porous coordination polymers. Porous coordination polymers (PCPs) are micro porous materials or coordination polymers that have large surface area grouped into flexible porous coordination polymers and rigid porous coordination polymers. Flexible porous coordination polymers are coordination polymers whose structures are transformable depending on external stimuli. They are highly flexible, porous with high surface area and possibility of rotation of their bridging ligands. Rigid porous coordination polymers are rigid and their structures are not transformable unlike the flexible porous coordination polymers [7].

Coordination polymers can also be classified according to dimensionality in which the structure can be determined to be one dimensional structure coordination polymer, two dimensional structure coordination polymers and three dimensional structure coordination polymers [8]. This classification depends on the number of directions in space the array extends. One dimensional structure coordination polymers extends in a straight line that is along the $\mathrm{x}$ axis as illustrated in Figure 1. Two dimensional structure coordination polymers extends in a plane that is in two directions, $\mathrm{x}$ and $\mathrm{y}$ axis illustrated in Figure 2. Three dimensional structure coordination polymers extends in all three directions, that is $\mathrm{x}, \mathrm{y}, \mathrm{z}$ axis as illustrated in Figure 3.

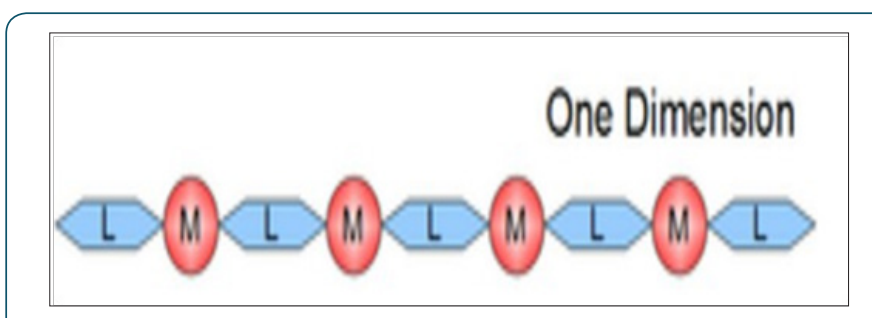

Figure 1: One dimensional structure coordination polymers.

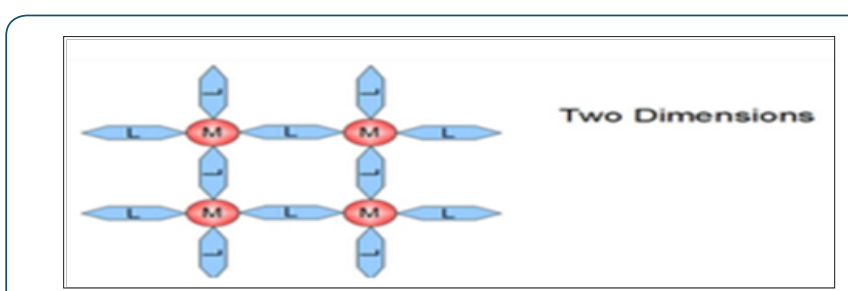

Figure 2: Two dimensional structure coordination polymers.

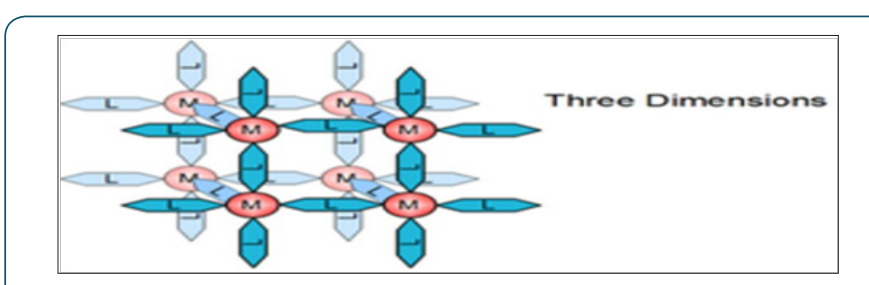

Figure 3: Three dimensional structure coordination polymers.

Factors that determine the crystal structure and dimensionality of coordination polymers

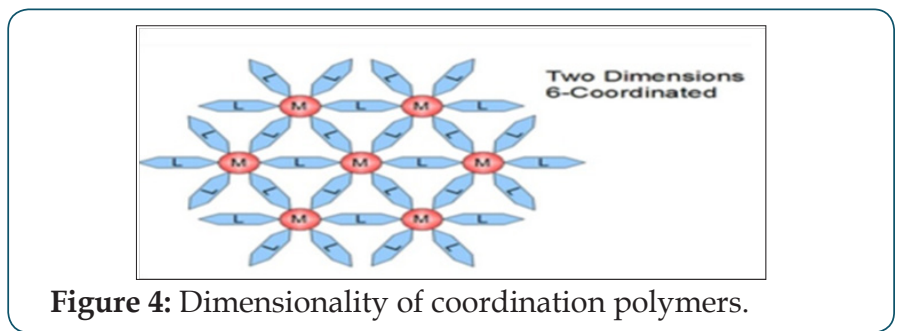

The crystal structure and dimensionality of the coordination polymer is determined by intermolecular forces and bonding, coordination geometry, metal centres, ligands, crystallization environment, counter ion and guest molecules. Intermolecular forces are one of the factors that affect the structure and dimensionalities of coordination polymers and include van der waal forces, $\pi-\pi$ interactions, hydrogen bonding and stabilization of $\pi$ bonds of polarization bonds in addition to the coordination bond formed between the metal and the ligand. The coordination geometry and the functionality of the linker of the metal center determine the crystal structure and dimensionality of the coordination polymers. Dimensionality as shown in Figure 4 is driven by the metal center that can possess the ability to bond to as many as sixteen functional 
sites on linkers. This number of possible bonds is the coordination number, commonly between 2 and 10 and the bonding sites are distinctly separated by angles [9].

Metal centers known as nodes or hubs bond to a particular number of linkers at well-defined angles. These number of linkers bound to a node is called coordination number and are held along with the angles which determine the dimensionality of the structure. Coordination number and coordination geometry of a metal center is determined definitely by the number of uniform distribution which increases with increase in cation size [9]. Prediction and explanation of coordination geometry is difficult in part due to the complex effect of environment on electron density distribution. Although, models like hybridization model and molecular orbital theory use Schrodinger equation to predict and explain it. Metal centers in coordination polymers could be that of

a) Transition metals: These are commonly used as nodes either in the atom or ion and particularly filled d-orbitals can hybridize differently. This depends on the environment of the transition metals, particularly copper and gold ions that have fully filled d-orbitals in their outer shell as neutral atoms exhibit multiple coordination geometries because of their electronic structure.

b) Lanthanides: These are large atoms that have coordination number varying from 7 to 10 . Lanthanides coordination environment are difficult to predict making them hard to use as nodes. They create avenue of mixing luminescent components.

c) Alkali metals and alkaline earth metals: These metals exist as stable cations and readily form cations with stable valence shells. This gives them different coordination behavior than transition metals and lanthanides. They are very much affected by the counter ion from the salt used in synthesis and that is hard to avoid. Consequently, the dimensionalities of the structures increase as the radius of the metal increase down the group that is, from calcium to strontium to barium as shown in Figure 5a-5c. These metal cations from the group 2 of the periodic table increase in metal cation size and polarizability leading to increase in dimensionality $[10,11]$.

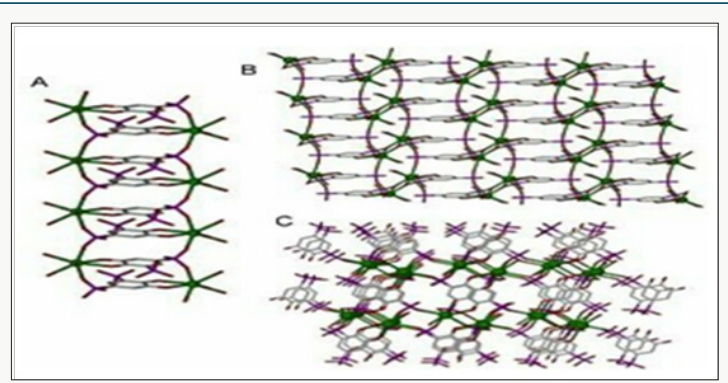

Figure 5a-5c: Diagrams showing (a) $[\mathrm{CaCl})(\mathrm{H} 2 \mathrm{O})] \cdot \mathrm{H}_{2} \mathrm{O}(\mathrm{b})$ $\left[\mathrm{Sr}(\mathrm{L})\left(\mathrm{H}_{2} \mathrm{O}\right) 4\right](\mathrm{c})\left[\mathrm{Ba}(\mathrm{L})\left(\mathrm{H}_{2} \mathrm{O}\right) 4\right] \cdot \mathrm{H}_{2} \mathrm{O}$.

A ligand in most coordination polymers will formally donate a lone pair of electrons to a metal cation and form a coordination complex by the means of acid/base relationship. Coordination polymer is formed by a ligand when it has the capability to form multiple coordination bonds and acts as a bridge between multiple metal centers. Ligands are of two types namely monodentate ligand and polydentate ligand which forms single coordination bond, referred to as terminal due to their inability to offer a place for the network to continue and form multiple coordination bonds that could lead to coordination polymers as they connect multiple metal centers together that an infinite array is formed respectively. These ligands can also form chelates, that is, multiple bonds of the same metal. Most at times, coordination polymers will contain a combination of polydentate and monodentate bridging, chelating and terminal ligands but is dependent on chemical composition and structural orientation [12]. Nearly atoms of any type that have a lone pair of electrons can be incorporated into a ligand.

Common ligands in coordination polymers include; polypyridines, phenanthrolines, hydroxyquinolines and polycarboxylates. Common binding sites are oxygen and nitrogen atom through other atoms like sulphur and phosphorous. As a consequence, the hard soft acid base theory (HSAB) trends are likely followed by ligands and metal cations. This implies that larger, more polarizable soft metals will coordinate more readily with larger more polarizable soft ligands and small, non-polarizable hard metals coordinate to small, non-polarizable hard ligands. Another factor that affects the structure of coordination polymers is the structural orientation. Ligands can either be flexible or rigid. A rigid ligand has no freedom to rotate around bonds or orient again within a structure. Flexible ligands produce more variety in the structure because they can bend, rotate around bonds and orient themselves again. There are examples of coordination polymers with two configuration of the same ligand within one structure [13] and two separate structures with different ligand orientation. Example of a flexible ligand is shown in Figures $6 a$ and $b$.
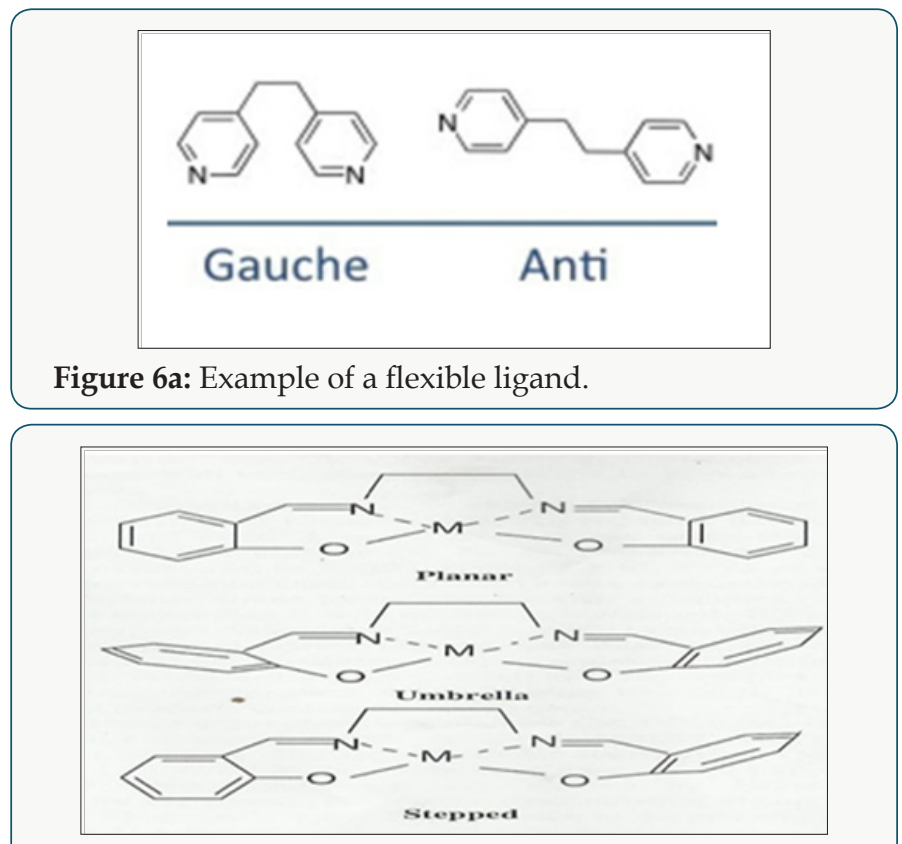

Figure 6b: Planar, umbrella and stepped molecular conformations of Salen complexes. 
Apart from metal and ligand choice, the structure of the coordination polymers can be affected by many other factors like counter ion. For instance, most of the metal centers are positively charged ions that exist as salts. A counter ion in the salt can affect the overall structure. Using silver salts as an example, AgNO3, AgBF4, AgClO4, AgPF6, AgASF6 and AgSbF are all crystallized with the same ligand. Their structures differ in term of the coordination environment of the metal and the dimensionality of the entire coordination polymer [14]. Crystallization environment also affects the structure of coordination polymer. Varying the crystallization environment can change the structure of coordination polymer. According to Ni et al. [15], changes in pH change the resulting structure of coordination polymer, also exposure to light or change in temperature do affect the structure [16]. However, these influences can be determined on a case by case basis. Guest molecules are molecules used to occupy pores or channels in coordination polymers. These molecules determine the stability of coordination polymer structure. Coordination polymer structures always have empty space in the form of pores or channels.

Thermodynamically, this empty space is not favorable. Therefore, in order to maintain a stable structure and prevent the collapse of coordination polymer structure, the channels or pores are often filled with guest molecules. These molecules do not form bond with the surrounding lattice but interact sometimes by the means of intermolecular forces like hydrogen bonding or $\pi$ stacking. Frequently, the solvent used in crystallizing coordination polymer will be the guest molecule but, it can also be anything like other salts present, atmospheric gases such as Nitrogen, Oxygen, CO2, etc. Lastly, guest molecule present can sometimes influence the structure of coordination polymers by supporting a pore or channels where otherwise none would exist. The structure of coordination polymer may be affected by the addition and removal of guest molecules. This is illustrated below in Figure 7a with (top) change of a linear 1D chain to a zigzag pattern, (middle) staggered 2D sheets to stacked, and (bottom) 3D cubes become more widely spaced. Where a lattice traps or contains guest molecules extending into polymeric species, a clathrate compound is formed. This polymeric compound envelops the guest molecule with bars or in lattice as illustrated in Figure $7 \mathrm{~b}$.

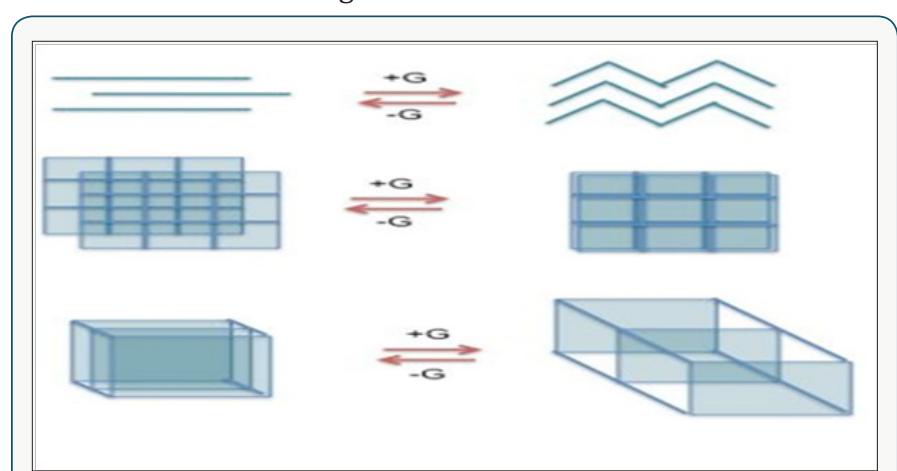

Figure 7a: Conformational changes in coordination polymers due to guest-host interaction.

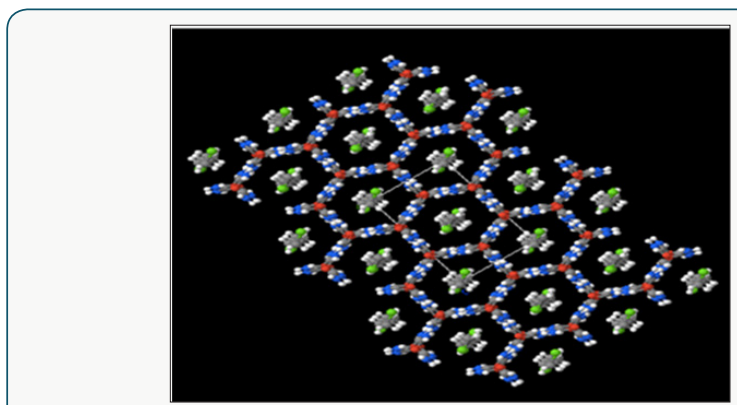

Figure 7b: Clathrate compound.

\section{Synthesis of coordination polymers}

Self-assembly which involves crystallization of metal salts with ligands, a replica of the method of growing crystals has been the commonest, reliable and age long method for synthesizing coordination polymers. These methods involve growing crystals of adequate size and quality as the characterization method involves the use of x-ray crystallography. Commonly used methods include slow diffusion (solvent layering), slow evapouration and slow cooling. Apart from this general method, some other methods applied in the preparation of some coordination polymers in order to get advantageous properties are discussed. The various methods, advantages and disadvantages are briefly listed. In slow evaporation method, metal salts and organic linkers are mixed together in the liquid phase with or without the aid of additional auxiliary molecules however, time-consuming is one of the major disadvantages in comparison to other conventional synthesis methods [17]. This could be overcome by using low-boiling solvents. Hydro/solvothermal method involves liquid-phase synthesis and the reaction is carried out at higher temperatures (temperature higher than 1000C) and pressure for several hours or days with the aid of closed vessels.

The method is advantageous because it offers high solubility of the precursors and the formation of good quality metal organic framework crystals suitable for structural characterization. On the other hand, microwave assisted synthesis which makes use of energy in the form microwaves for a period of about an hour is advantageous because the technique is adjustable to power outputs, pressure and a wide range of temperatures. Other methods such as mechanochemical method are considered better because the use of organic solvents can be avoided, room temperature is sufficient, side products formed are harmless and MOF can be obtained in short reaction time [17]. In sonochemical synthesis, the idea is to shorten the crystallization time by the application of ultrasound energy thereby creating energy efficient, environmental friendly method used to generate homogeneous nucleation centers which has no direct interaction between ultrasound and molecules. The emergence of electrochemical synthesis brings into focus a fast and continuous production of large amounts of metal organic framework crystals with the use of metal ions in synthesis instead of metal salts. 


\section{Synthetic methods of transition metal coordination polymers}

Transition-metal coordination polymers are generally prepared using three general methods. These methods include the following; use of ligands capable of simultaneously coordinating to two metal atoms to generate the desired polymer, use of performed polymers to which metal atoms are attached to yield polymers of useful properties and the use of transition-metal coordination complexes containing at least one of the ligands with functional group useful in polymer formation [18,19]. The use of ligands capable of simultaneously coordinating to two metal atoms to generate the desired polymer could be represented using equation 1 .

$\mathrm{nM}+\mathrm{n}(-\mathrm{Y}-) \rightarrow(-\mathrm{M}-\mathrm{Y}-) \mathrm{n} 1$

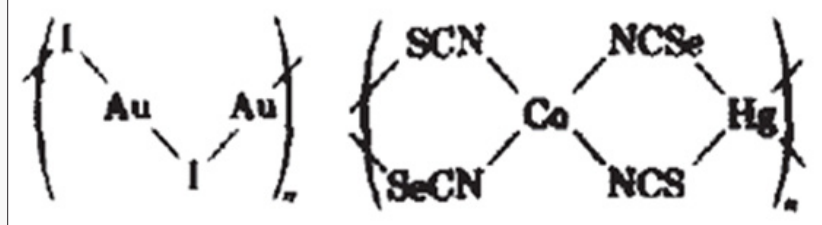

Figure 8a: Coordinating polymer formed from monodentate halide or pseudohalide ligands.

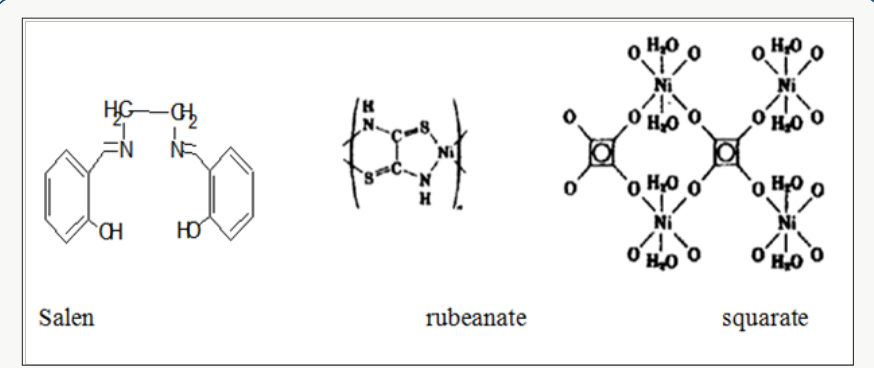

Figure 8b: Coordinating polymer formed from bis-chelating ligands.

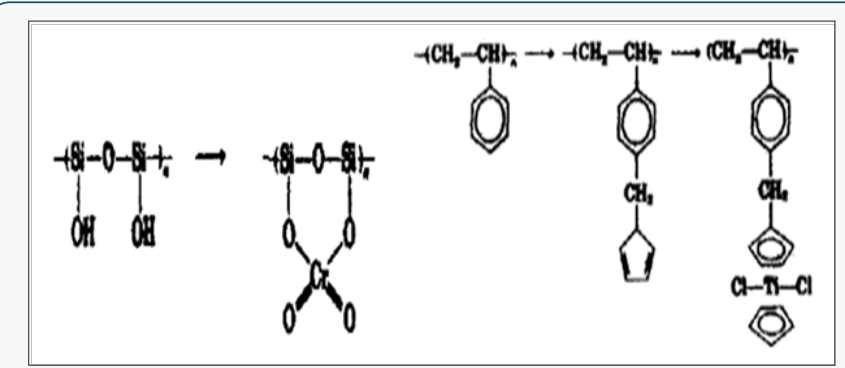

Figure 9: Silica and polystyrene support polymer.

$\mathrm{M}$ the metal atom and $\mathrm{Y}$ the ligand. For a monodentate halide or pseudohalide ligands, coordinating polymer illustrated in Figure $8 \mathrm{a}$ is formed. In a case where the ligand is multidentate, structures such as that in Figure $8 \mathrm{~b}$ could be formed from bis-chelating agents like Salen, rubeanate or squarate. Grubbs et al. [20] noted that silica and polystyrene support polymer with phosphines, amines or cyclopentadienyl ligands act as functional group on polystyrene supports as illustrated in Figure 9. Recently, the use of transitionmetal coordination complexes in which at least one of the ligands has functional group that can be used in polymer formation is on the increase. Much interest has been on this method in recent years and substituted metallocenes have been the most interesting in this research. Pittman et al. [21] observed that vinylferrocene forms both homo- and copolymers on polymerization in almost the same pattern as ethylene polymerizes by addition as illustrated in Figure 10a.

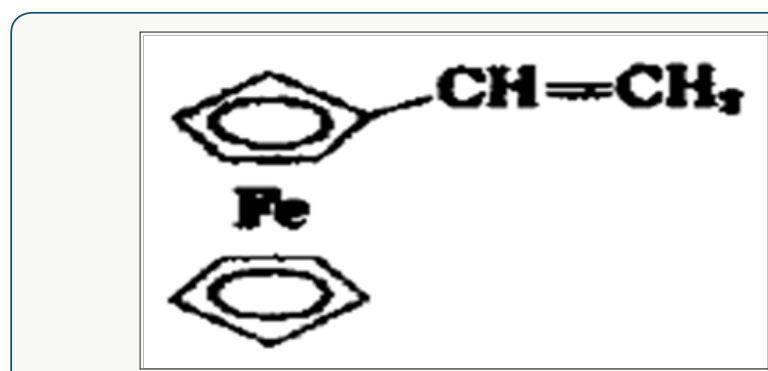

Figure 10a: Vinylferrocene coordination polymer.

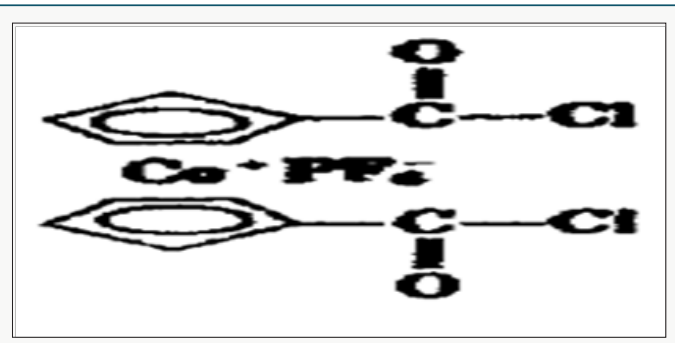

Figure 10b: Polyesters formation between metallocene and alkanols.
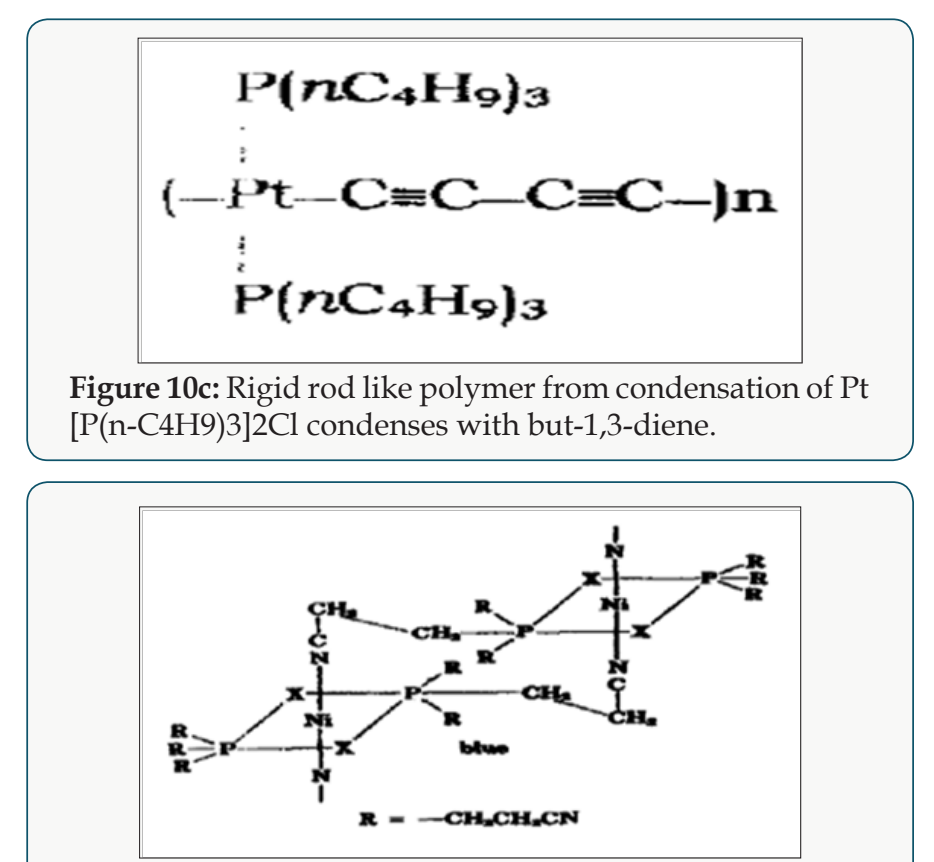

Figure 10d: Linear chains of octahedral coordination.

In a similar faction, acid chlorides of various metallocenes undergo condensation with alkanols to form polyesters as shown in Figure 10b. Condensation complexes such as, Pt [P (n-C4H9)3]2Cl condenses with but-1,3-diene to form rigid rod like polymer illustrated in Figure 10c. Foxman \& Janfmann [22] showed that 
square planar $\mathrm{NiX} 2[\mathrm{P}(\mathrm{CH} 2 \mathrm{CH} 2 \mathrm{CN}) 3] 2(\mathrm{X}=\mathrm{Cl}, \mathrm{Br})$ complexes polymerize either in solution or solid state to form linear chains of octahedral coordination as the nitrogen atom of one cyanoethyl chain bonds to the nickel atom of another adjacent complex as shown in Figure 10d.

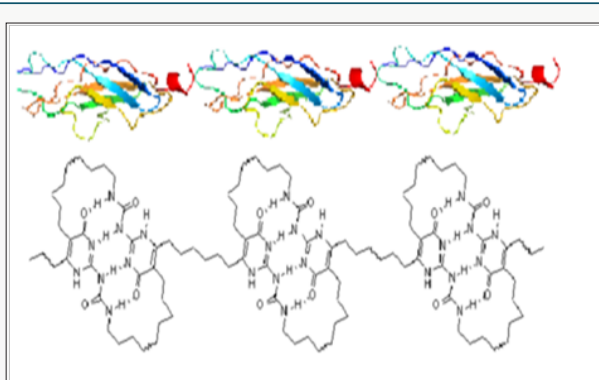

Figure 10e: Example of supramolecular polymer.

\section{Synthetic method of porous coordination polymers}

Generally, porous coordination polymers (PCPs) are prepared in the liquid phase by using solvent as a medium to induce the self-assembly of a regular framework. This type of reaction can be carried out by mixing the metal ion solution with the chelating ligand solution at room temperature or under hydrothermal or solvothermal conditions [23]. Metal ion and ligand, solvent and counter ions must be involved to get porous coordination polymers of desired property. The limitation of flexible ligand products complexes of been structurally amorphous therefore allows the use of rigid or semi-rigid ligands for such purposes. The rigidity of aromatic ligands which may be neutral, cationic and anionic other than the aliphatic ones gives them an edge in the synthesis. Initially, the prepared porous coordination polymers do not have void space because the guests filled all the cavities and these guests may be solvent molecules, excess ligands or counter ions. To make the void spaces usable, the guest molecules are substituted with sufficiently volatile or exchangeable solvent molecules.

Notwithstanding the relevance of vacant spaces, some porous coordination polymers cannot exist without the guest molecule. In some porous coordination polymers the solvents collapses after and are removed. In such a case, the solvent are likely to be unimportant in the use of the polymer as it will be eliminated after the preparation of the polymer. Solvent play notable role in the preparation and characteristics of porous coordination polymers such as the size and shape of pore and can be controlled by using different solvents [24]. The evolution of another branch of coordination polymers, the supramolecular polymers as shown in Figure 10e. The monomeric units are held together through highly directional and reversible non-covalent bonding relationship which subsequently defines their properties. Similarly, building frame work template and formation of open molecular structure is feasible eliminating catenation. Sareeya et al. [24] demonstrated notable examples such as (a) $\left[\left(\mathrm{Co}_{3} \text { (ndc) }\right)_{3}\right.$ (bipyen)1.5] $\left.\cdot \mathrm{H}_{2} \mathrm{O}\right] \mathrm{n}$ and (b) $\left[\left(\mathrm{Co}_{3}\right.\right.$ (ndc) (bipyen)1.5] $\cdot \mathrm{H}_{2} \mathrm{O}_{\mathrm{C}} \mathrm{H}_{6}$ ]n where ndc is naphthalinedicarboxylate as shown in Figures 11a \& 11b.

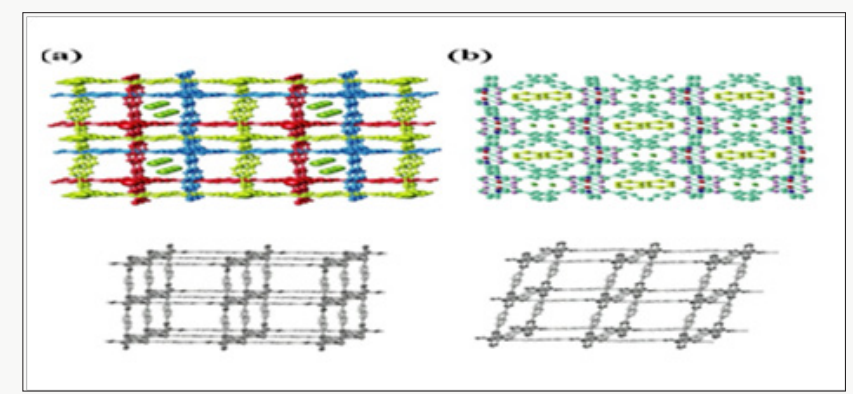

Figure 11(a,b): Structures of (a) $\left[\left(\mathrm{Co}_{3}(\mathrm{ndc})_{3}\right.\right.$ (bipyen)1.5]. $\left.\mathrm{H}_{2} \mathrm{O}\right] \mathrm{n}$ and (b) $\left[\left(\mathrm{Co}_{3}(\mathrm{ndc})\right.\right.$ (bipyen)1.5]. $\left.\mathrm{H}_{2} \mathrm{O} \cdot \mathrm{C} 6 \mathrm{H}_{6}\right] \mathrm{n}$ where ndc is naphthalinedicarboxylate (Sareeya et al, (2008)).

Specifically, Cd(NO3)2(1,2-bis(4-pyridyl)ethyne)1.5]n was made by the reaction of $\mathrm{Cd}(\mathrm{NO} 3) 2.4 \mathrm{H} 2 \mathrm{O}$ with trans-1,2-bis(4pyridyl)ethyne in a methylene chloride/methanol solvent system. Large pale yellow infinite one dimensional structure of inter penetrating molecular ladder coordination polymer crystals formed at the interface of methanol/methylene chloride was collected by filtration to give a yield of $50 \%$ as shown in Figure 12. Similarly, [[Cd(NO3)2(1,2-bis(4-pyridyl)ethene)1.5]n was synthesized from the reaction of $\mathrm{Cd}(\mathrm{NO} 3) 2.4 \mathrm{H} 20$ with the trans1,2-bis (4-pyridyl)ethene ligands in ethanol/water system. At the interface of the ethanol/water large colorless crystals were formed, collected by filtration and washed severally with n-hexane. The coordination polymer formed of $75 \%$ yield has a novel infinite noninterpenetrating zigzag chain structure that is made up of trans-1, 2-bis (4-pyridyl)ethene-Cd-trans-1,2-bis(4-pyridyl)ethene-Cdtrans-1,2-bis(4-pyridyl)ethene building units as shown in Figure 13.

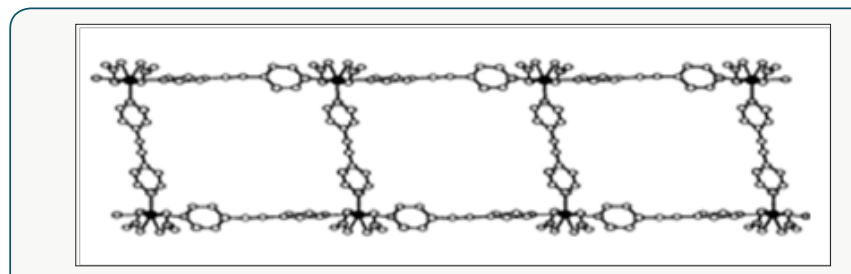

Figure 12: Coordination environment around the cadmium atom in [Cd(NO3)2(1,2-bis(4-pyridyl)ethyne)1.5]n (Sareeya et al, (2008).

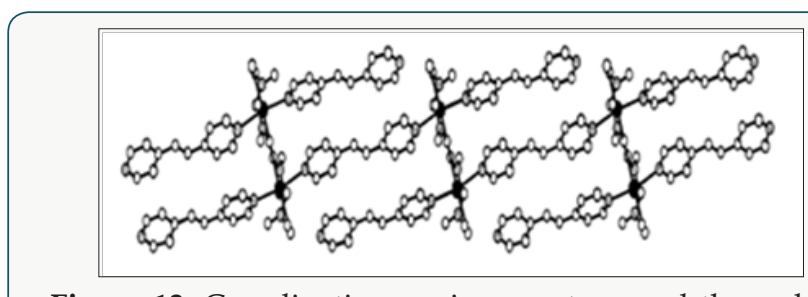

Figure 13: Coordination environment around the cadmium atoms in [Cd(NO3)2)2(1,2-bis(4-pyridyl)ethene)1.5]n ( Sareeya et al, (2008).

[Cd( $\left.\mathrm{NO}_{3}\right) 2$ (1,2-bis(4-pyridyl)ethane)1.5]n was also synthesized by the reaction of $\mathrm{Cd}(\mathrm{NO} 3) 2.4 \mathrm{H} 20$ with the 1,2-bis (4-pyridyl) 
ethane chelating ligand in a methylene chloride/methanol system. Colourless crystals were obtained overnight, collected by filtration with a yield of $72 \%$. This coordination polymer forms square units that are connected through the two Cd (II) centers to give infinite linear chains [25] as shown in Figure 14.

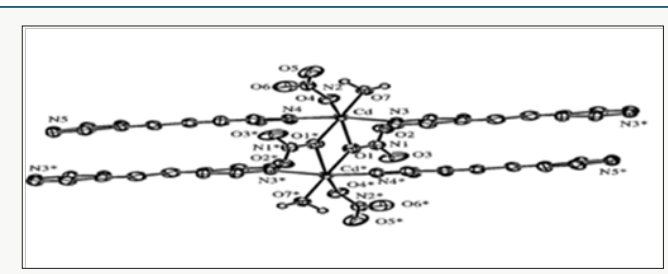

Figure 14: Coordination environment around the cadmium atoms in [Cd(NO3)2)2(1,2-bis(4-pyridyl)ethane)1.5]n ( Sareeya et al, (2008)).

\section{Nanoporous coordination polymers}

Coordination polymers have been synthesized and used for a wide range of applications including gas storage, nonlinear optics, drug delivery, catalysis and chemical sensing but recent works has been extended to templates synthesis of silica nanoshells nanoporous carbon and metallic nanoparticles [1] bearing in mind that most nanoparticles contain either only organic or only inorganic components. A typical template is represented in Figure 15. Many methods abounds for the synthesis of nanoparticles of coordination polymers or nanoparticles of metal organic frameworks but all of the processes takes advantage of reduced solubility of the particles in comparison of the individual components. Common methods rountinely used include solvothermal synthesis, simple mixing of precursor solutions, precipitation by rapid addition of a poor solvent, high-temperature surfactant-assisted synthesis and reverse microemulsion synthesis $[1,2,26]$. Specifically, a nanoparticle metal organic framework with the formula $\mathrm{Zr}_{6} \mathrm{O}_{6}(\mathrm{OH})_{4}(\mathrm{BDC})$ (BDC = 1,4-benzenedicarboxylate) was made by the solvothermal method.

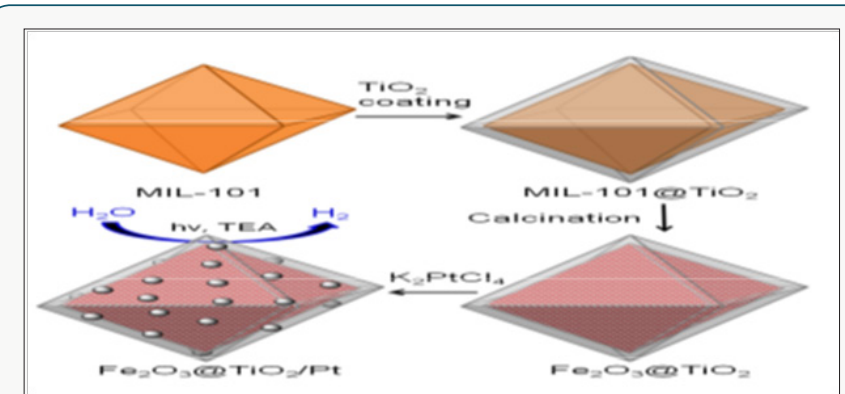

Figure 15: Coordination polymer templated synthesis of Fe2O3@TiO2 by coating MIL-101 with TiO2 followed by calcination, and its use for photocatalytic hydrogen production after depositing Pt particles (Kitagawa et al., 2004).

Another group of coordination polymers regarded as cyanometallates is composed of relevant building blocks for series of one, two and three dimensional coordination polymeric networked structures with transition metal templates. Some authors [27-29] noted that this group of strikingly important compound possesses clathrate hosts' structure with properties not limited to spin-crossover phenomena and molecular magnetism. In a typical synthesis, Dursun et al. [30] prepared $\left(\mathrm{K}_{2}\left(\mathrm{Ni}(\mathrm{CN})_{4}\right] \cdot \mathrm{H}_{2} \mathrm{O}\right)$ cyanometallate by mixing the stoichiometric amounts of $\mathrm{NiCl}_{2}$. $6 \mathrm{H}_{2} \mathrm{O}$ with potassium cyanide in water. The coordination polymer $\left[\mathrm{Cd}\left(\mathrm{NH}_{3}\right)(\mu\right.$-ampy) $\mathrm{Ni}(\mu$-CN)4]n (ampy= 3-aminomethylpyridine) was synthesized by mixing together with the water $\mathrm{K}_{2}\left(\mathrm{Ni}(\mathrm{CN})_{4}\right]$. $\mathrm{H}_{2} \mathrm{O}$ and cadmium compound of 3-aminomethylpyridine. The coordination polymer of 3D conformation crystallizes in the orthorhombic crystal system with the asymmetric unit containing one $\mathrm{Cd}(\mathrm{II})$ ion, one $\mathrm{Ni}(\mathrm{II})$ ion, one amine, four cyano and bis(monodentate) bridging ampy ligands having Ni(II)ion coordinated in square-pyramidal geometry as illustrated in Figure 16.

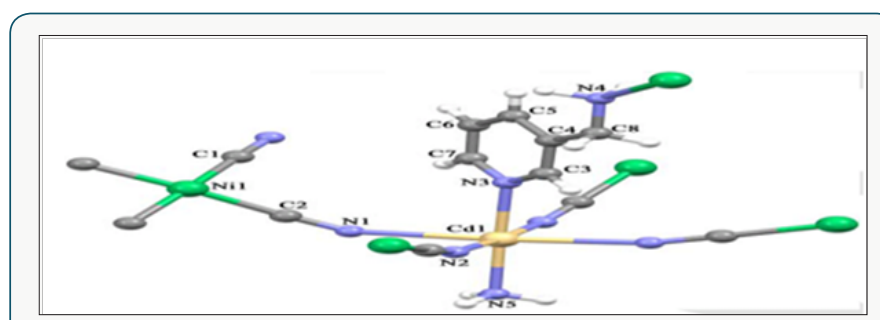

Figure 16: Coordination polymer templated synthesis of Fe2O3@TiO2 by coating MIL-101 with TiO2 followed by calcination, and its use for photocatalytic hydrogen production after depositing Pt particles (Kitagawa et al., 2004).

\section{Properties of Coordination Polymers}

Notable unique properties attributed to coordination polymers makes these groups of compounds very useful in various applications. This has increased research in coordination polymers where transition metals are linked with suitable ligands in the past years. Notable excellent properties not limited to semiconducting and catalytic properties, protective coating properties, waste water treatment for metal recovery, as antifouling paints and anti-microbial properties. Coordination polymers with efficient ionophores have been of much interest and constant consideration owing to their efficient therapeutic and fluorescence properties $[31,32]$. Some coordination polymers containing ionosphores of 8-Hydroxyquinoline are important in areas of metal recovery from waste water as they exhibit complexing ability, as water disinfectants, protective coatings, ion-exchange resin, gels and ointment for medical applications, antifouling paints, antimicrobial agents, surgical materials.

\section{Electrical conductivity}

Coordination polymers exhibit electrical conductivity as one of their important properties. Some coordination polymers have short inorganic and conjugate organic bridges in their structure which provide pathways for electrical conduction. A one dimensional coordination polymer that exhibit conductivities in a range of $1 \times 10$ 6 to $2 \times 10-1 \mathrm{~S} / \mathrm{cm}$ is shown in Figure 17 . The conductivity has been supported to be as the result of the interaction between the metal $d$-orbital and the $\pi$ level of the bridging ligand [2]. Coordination polymers could also exhibit semi-conductive property and a typical 
example is the three dimensional structures that comprise sheets of silver containing polymers when the metal centers are aligned. The conduction decreases as the silver atoms move from parallel to perpendicular direction.

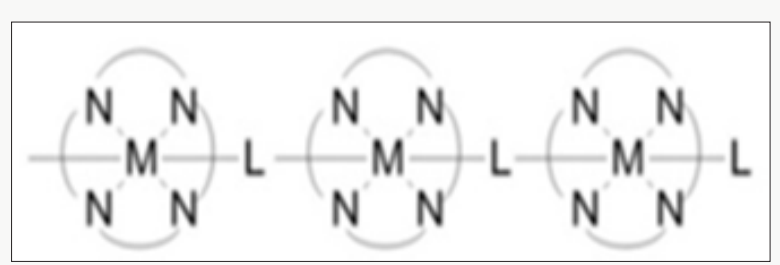

Figure 17: One dimensional coordination polymer where $\mathrm{M}=\mathrm{Fe}, \mathrm{Ru}, \mathrm{OS} ; \mathrm{L}=$ Octaethylporphyrinato or pthalocyaninato and $\mathrm{N}$ belongs to pyrazine or bipyridine

\section{Magnetism}

Magnetism is one of the major properties exhibit by coordination polymers. They show three types of magnetism a consequence of cooperative phenomena of the magnetic spins within a solid and which include antiferromagnetism, ferromagnetism and Ferrimagnetisms. The property results from the coupling between the spins of the paramagnetic centers. Research [2,33] has shown that efficient magnetic property is obtained when metal ion is bridged by small ligands thereby allowing short metal- metal contacts as exemplified in cyano, or oxo, azido bridges.

\section{Luminescence}

Luminescent coordination polymers typically contain organic chromophoric ligands that absorb light and then transmit the excitation energy to the metal ion. Coordination polymers are the most versatile, efficient and effective luminescent species potentially because of their emission properties being couples with guest exchange relationship [2,34]. Of recent, luminescent supramolecular architecture has been of much interest and great deal of work has been on the high gear on their potential applications in optoelectronic devices or as fluorescent sensor and probes since they are stable, both thermo and solvent resistant in comparison to organic species. Katz et al. [35] has noted that some ligands fluoresce in the absence of metal linker not because of ligand to metal charge transfer but the intense photoluminescence emission of the coordination polymers is of magnitudes higher than that of the free ligand. This further explained the fact that increase in rigidity and asymmetry of the ligand upon coordination to the metal center increases the fluorescence of the specie.

\section{Sensor capability}

Of notable interest in the property of coordination polymers is the change in colour upon the change of solvent molecule mixed into the guest structure. The variation or replacement of the solvent in the coordination sphere leads to change in the geometry of the polymer necessary in sensor application. A typical example when two cobalt coordination polymers of the type $[\operatorname{ReS}(\mathrm{CN})] 4-$ - cluster contains water ligands coordinated to the cobalt atoms. Replacement of water with tetrahydrofuran changes the original orange solutions to either purple or green while on addition of diethylether, the colour changes to blue $[2,36]$. Coordination polymers in this case act as solvent sensor capable of physically changing color in the presence of certain incoming solvents displacing the water ligands on the cobalt atoms resulting in a change of their geometry from octahedral to tetrahedral.

\section{Non-linear optical behavior}

Non-linear optical behavior one of the striking properties of coordination polymers describes the ability of a material or materials to convert frequency and intensity modulation. Research $[34,37]$ has shown that one of the best known non-linear optical materials is a one dimensional coordination polymer, potassium titanyl phosphate (KTP). KTP characteristically has high non-linear optical co-efficient, low threshold power, high optical damage threshold and low phase matching sensitivity [37] and could be a good replacement for silicon solar cells which is limited in mobile applications and processing cost.

\section{Flexibility}

This very unique property peculiar to third generation porous coordination polymers because they showcase flexible framework and dynamic functionalities is highly sought in industrial polymer materials. Sareeya et al. [7] noted that this form of flexibility common to porous coordination polymers and not observed in other porous material like zeolite and activated carbon makes the coordination polymers respond to external stimuli by reversible structural transformation. Thus, the guest molecules serve as the stimuli capable of transformation of flexible porous coordination polymers with the rotation of the bridging ligands possible due to uncommon high flexibility of the coordination polymer. The presence of high void space provides more applicable and efficient function for this group of compounds as they are more dynamic than other non-porous solids as enough space allows ligand rotation. Typically, a 3-dimensional porous coordination polymer [(Zn3(nbt)2(EtOH)].4EtOH]n (Figure 18) shows reversible structural transformation through the coordination geometry of $\mathrm{Zn} 2+$ changing from a trigonal bipyramide [(Zn3(ntb)2(EtOH)2] $\mathrm{n}$ to a tetrahedral [Zn3(ntb)2]n after the removal of EtOH molecules coordinated to the framework without the collapsing the structure. Similarly, rotation of the O-C-O plane of the carboxylate part geometrically changes the structure. (ntb $=\mathrm{NN}, \mathrm{N}^{\prime}, \mathrm{N}^{\prime \prime}$ nitrilotrisbenzoate).

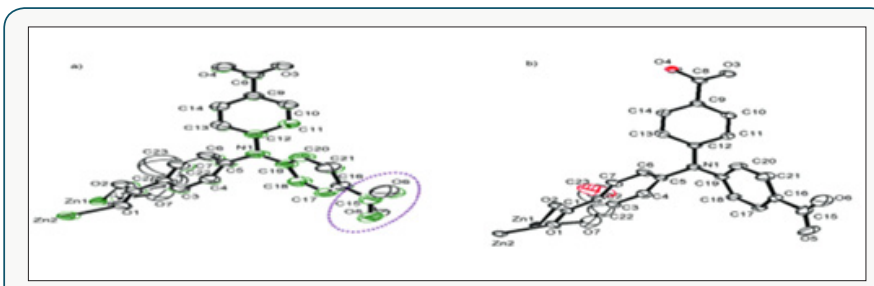

Figure 18: Structures of (a): [( $\left.\mathrm{Zn}_{3}(\mathrm{ntb})_{2}(\mathrm{EtOH})_{2}\right] \mathrm{n}$ to a tetrahedral $(b)$ : $\left[\mathrm{Zn}_{3}(\mathrm{ntb})_{2}\right] \mathrm{n}$ where $\left(\mathrm{ntb}=\mathrm{NN}, \mathrm{N}^{\prime}, \mathrm{N}^{\prime \prime}\right.$-nitrilotrisbenzoate) (Sareeya et al 2008). 


\section{Thermal stability}

Thermal stability of coordination polymers is one of the most striking properties of this group of compounds. They are notably of low thermal conductivity, low density and exhibit phonon phenomena (energy carriers are linked with the vibration modes of crystal lattices) making them dielectric materials [2,7]. The thermal conductivity of porous coordination polymers rely on the mode of phonon scattering which could be phonon-grain boundary, scattering defect-phonon scattering or phonon scattering. Recently research by Huang et al. [38] opined that thermal conductivity of single crystal metal organic framework below $35 \mathrm{~K}$ of temperature increases with temperature increase and defect phonon scattering mechanism dominates. Within the range of 35 to $100 \mathrm{~K}$ of temperature, phonon-phonon scattering mechanism becomes prominent leading to rapid thermal conductivity decrease with temperature increase whereas above $100 \mathrm{~K}$ of temperature, thermal conductivity is mostly independent of temperature indicating minimum phonon mean free path.

\section{Porosity}

Sareeya et al. [7] noted that porous coordination polymers are highly porous and they have high surface area with permanent pore size and pores shape. This is an advantage of porous coordination polymers for gas storage application. This property has given them edge over their non- porous counterpart in industrial applications.

\section{Solvent resistance and ferroelectric property}

Coordination polymers are resistant to solvents compared with purely organic species [2]. Coordination polymers especially the Shiskabob class of transition-metal coordination polymers [39] of typical example the non-planar metalloporphyrins with non-symmetrical bridging ligands carry a net and aligned dipole moment along the stacking axes as shown in Figure 19. This dipole moment originate from the change separation between the bowl shaped porphyrinato core and the metal atom. The metal atom tends to be pulled out of the porphyrin plane by a stronger axial ligand.

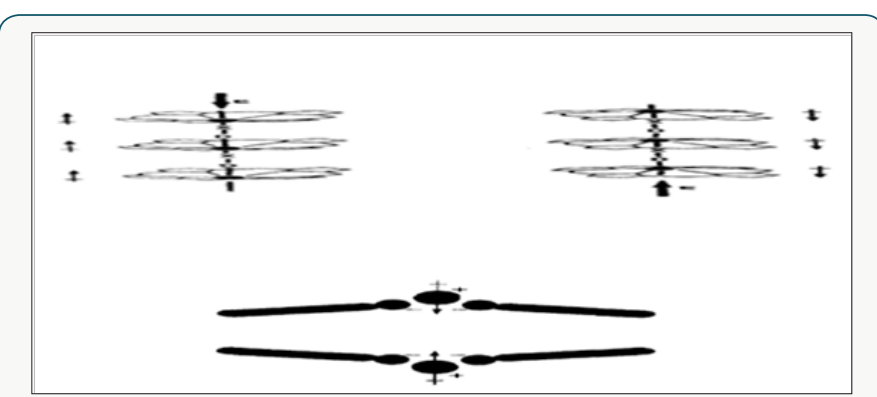

Figure 19: The schematic representation of ferroelectric coordination polymer and dipole moment switching in response to an external field.

\section{Applications of Coordination Polymers}

Coordination polymer an organometallic or inorganic polymer or a coordination compound with coordination complexes as repeating units are important in inorganic and organic chemistry, material science, pharmacology and electrochemistry because of the stunning variety of their structure, compositions and easy tailoring widening their applications in most areas of modern society $[7,40,41]$. Specifically, they are used as commercial dyes, in separation of hydrocarbons, as molecular sieves, light emitting diodes and other miscellaneous applications.

\section{Pigment}

Coordination polymers are used mainly in the commercial form as dyes. A cyanide complex known as Prussian blue $\left(\mathrm{Fe}_{4}\left[\mathrm{Fe}(\mathrm{CN})_{6}\right]\right.$. $\mathrm{XH} 2 \mathrm{O}$ ) is also commercially used as dye. The compound is easy to make, cheap, non-toxic and intensely colored and widely used as a pigment in oil and water color and dyeing as pigments in the production of black and bluish inks and a common pigment in paint [7].

\section{Separation of hydrocarbons}

Among the early commercialized coordination polymers are the Hofmann compounds which crystallize with small aromatic guests such as some xylenes, benzene and of the formula $\mathrm{Ni}(\mathrm{CN})$ $\mathrm{Ni}(\mathrm{NH})$ [2]. The selectivity of this material has been applied in the separation of hydrocarbons.

\section{Electrical conductors and semiconductors}

This is one of the most important applications of coordination polymers. Many coordination polymers are used in materials science as electrical conductors and semiconductors. Metal conductivity is generally higher than semiconductors with temperature dependence defining the difference as increase in temperature increases electrical conductivity in metals unlike the semiconductors in which increase in temperature decreases their conductivity. The interest in superconducting materials is solely in synthetic metals and of interest are dimensional coordination polymers that conduct electricity. The best known are the cyano platinum salts $\left(\mathrm{Pt}(\mathrm{CN})_{4}\right] \mathrm{n}$ - and halocarbonyliridium salts $(\operatorname{Ir}(\mathrm{CO}) 2 \mathrm{Cl} 2] \mathrm{n}$-. which are cation deficient salts shorter than $2.9 \AA \AA$ and structurally shown as a and b respectively in Figure 20 (a) $\operatorname{Rb} 2[\mathrm{Pt}(\mathrm{CN}) 4][\mathrm{FHF}] 0.4$ and (b) $\mathrm{K} 0.58[\operatorname{Ir}(\mathrm{CO}) 2 \mathrm{Cl} 2$. Other ambient pressure superconductors based on electron transfer , metal -metal distance, periodical variation of the charge density along radical donor and polymeric anions in such compounds has electron transfer donor molecules alternating layers in their crystal structure and polymeric anions [37].

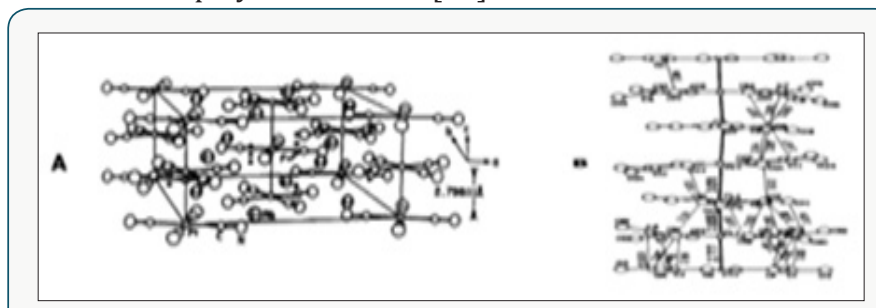

Figure 20: Structures of cation deficient salts of (a) $\mathrm{Rb}_{2}\left[\mathrm{Pt}(\mathrm{CN})_{4}\right][\mathrm{FHF}] 0.4$ and (b) $\mathrm{K} 0.58\left[\operatorname{Ir}(\mathrm{CO})_{2} \mathrm{Cl}_{2}\right.$. 


\section{Luminiscent materials}

Luminescent polymers are used as fluorescent sensors and probes because of the presence of organic chromophoric ligands which absorbs light and then pass the excitation energy to the metal ion. Potentially, coordination polymers are the most versatile luminescent species because of their emission properties together with guest exchange and are efficient materials for light emitting diodes $[2,35,37]$.

\section{Medicine}

The application of coordination polymers in medicine is currently on the rise with special emphasis on nanoparticle drug delivery. Photolytically sensitive caged compounds have been examined as containers for releasing a drug or reagent $[42,43]$. A metal organic framework with the formula Zr606(OH)4(BDC) (BDC $=1,4$-benzenedicarboxylate) was made by the solvothermal method. This nanoparticle metal organic framework is important in x-ray computed tomography (CT) a type of biomedical imaging that is capable of providing three-dimensional images with excellent spatial resolution. CT is mainly and recently used to image a wide range of structures within and throughout the whole body not limited to organs, blood vessels, and bones. The technique is a relevant tool for diagnosing and monitoring abnormal health conditions and ailments throughout the body with conditions such as tumors, calcifications, embolisms, aneurysms, and inflammation efficiently and effectively treated $[2,44,8]$. Similarly, one of the cyanide complexes, $\mathrm{Fe} 4[\mathrm{Fe}(\mathrm{CN}) 6] 3 . \mathrm{XH} 2 \mathrm{O}$ has the ability to incorporate mono-cations and this makes it an important separating agent for certain heavy metal poison including but not limited to patients who have ingested radioactive caesium or thallium.

\section{Laboratory histopathology stain for iron}

One of the recognized, common, honoured, effective and efficient coordination polymers used in histopathology stain to detect iron presence in biopsy specimens by pathologists in bone marrow samples is $\mathrm{Fe} 4[\mathrm{Fe}(\mathrm{CN}) 6] 3 . \mathrm{XH} 20$ [4]. The deposits of iron in the tissue form purple Prussian blue dye in place and are seen as blue or purple deposits.

\section{Gas storage and trapping}

Flexible porous coordination polymers have attracted attention as gas storage vessel recently because of their high porosity and surface area. Fuel gases like hydrogen ( $\mathrm{H} 2)$ and methane (CH4) due to their economic, environmental balanced benefits have been realized as vehicle fuels and have attracted much interest as replacements of petroleum and diesel power source. However, Kesanli et al. [45] have noted that inefficient storage, in other words, low mileage owning to their low volumetric density is a major disadvantage of fuel gas for automobile use. Storage of these gases in adsorbate phase has been observed to increase the volumetric density and this has been efficiently done at room temperature and pressure of 0-30 atm using porous coordination polymer [46]. In the same way, Zn40-based MOF compounds are well known for their application to fuel gas storage because of their high porosity and large surface area. Though, some of the MOFs are too large for effective adsorption of hydrogen due to presence of large void space, however the unused space increases the volumetric density and hence smaller pores are favorable but controllable by the use of nonvolatile guests.

The intercalation of nonvolatile guests in the porous coordination polymers decreases the void space and pore size making the active surface guests an adsorbent as well. Decrease in pore size is better probably because the interaction between the aromatic ring of the donor ligand and the fuel gas leads to increase in storage ability [7]. Recently, coordination polymers with pillared layers structures are applied in adsorption to tune the pore size to that of the adsorbed gas and are effective for stabilizing the explosive gases like acetylene. Since they are structurally transformable, pore shape and size can be adjusted towards the most stable state with guest to improve host -guest interaction. The molecular interaction between the acetylene molecules placed in porous coordination polymer and acetylene molecule and the wall of the flexible porous coordination polymers is reduced and the system is stabilized a consequence of the large surface area of the flexible porous coordination polymers. Typically, [Cu3(BTB)2(H2O)3. (DMF)9(H20)2]n where (BTB = 4,4I,4 I I -benzene-1,3,5-tribenzate) the so called MOF-14 shows high $\mathrm{H} 2$ uptake because of the large void space and the interpenetrating fashion [7].

\section{Selective adsorption and separation}

The world is becoming more complex with an ever increasing search for a more efficient, effective, energy-saving and environmentally friendly procedure for separation and adsorption of gases and this calls for the design of tailored structures with tunable surface properties. The flexible porous coordination polymers and metal organic frameworks are promising candidates as adsorbents for gas separations owing to their thermal stability, large surface areas, adjustable pore sizes and tunable properties $[47,48]$. Flexible porous coordination polymer shows unequalled high selective guest sorption even though interpenetration results in porosity reduction. The combination of the flexibility of flexible porous coordination polymer and functional surface activity enables effective selectivity. The flexible porous polymer $\{\mathrm{Ni}(\mathrm{bpe}) 2(\mathrm{~N}(\mathrm{CN}) 2]$ $(\mathrm{N}(\mathrm{CN}) 2\} \mathrm{n}$ where (bpe =1,2-bis (4-pyridyl)ethane and $\mathrm{N}(\mathrm{CN} 2)=$ dicyanamide) is a 2-fold interpenetrating frame work which shows selective gas adsorption and anion exchange property. The tunable, modifiable active site and dynamic ability of the flexible porous coordination polymers makes it effective in the adsorption of all sizes of gases [7].

\section{Nanoreactors for polymerization}

Current research is on the rise in the use of porous coordination polymers as nanoreactors for polymerization. The behavior, regularity, reactivity and nature of monomers could be affected by the interaction between the host framework and monomers as 
well as the restricted available nanospace. The result amounts to polymerization that is precisely controlled and as well the polymer arrangement. Researches $[7,49,50]$ has indicated that when one dimensional nanochannels of crystalline porous compounds are filled with polymerizable groups of guest molecules, the monomers can be polymerized as well. Sareeya et al. [7] has noted that the porous coordination polymer (Zn2(1,4-dbc)2(dabco]n where (bdc=benzenedicarboxylate, dabco=1,4-diazabicyclo[2.2.2] octane) is an important nanoreactor for polymerization because, the introduction of p-divinylbenzene(DVBs) into (Zn2(1,4dbc)2(dabco)n channel causes the lattice to expand small compared with the original host though without effect on the framework.

\section{Anaytical chemistry}

In analytical chemistry, coordination polymer is used in the determination of phenols. Specifically, one of the cyanide complexes (Fe4(Fe(CN)6)3.XH2O is formed in its assay for total phenols and very effective in the spectrophotometric study of phenols or polyphenols [51,52].

\section{Conclusion}

Coordination polymers, metal-organic frameworks, clathrates and cyanometallates represent an emerging class of hybrid inorganic-organic materials that have attracted the attention of solid-state chemists owing to their combined unprecedented degree of porosity with other notable functional properties that are predominantly active in the ligand or metal moiety. Their ease of synthesis, modifiable and tunable characteristics are second to none in science and engineering. These class of polymers have opened a new area of research because of their versatility, applicability, thermo stability and resistant to solvents. Highest known surface areas has been generated and shown by coordination polymers and metal organic frameworks with lowest crystal densities and thermal stabilities offering them as important materials for high hydrogen storage, selective heterogeneous catalysis, magnetic ordering channels for regulating polymerizations, selective carbon dioxide capture and guest-dependent luminescence, nanoparticles in medicine and drug delivery, non- linear optical devices among others. Thus, this new class of materials due to their diversity, multiplicity, and exceptional properties are important and required to solve some of our most pressing societal problems including but not limited to creation and deployment of clean, sustainable environment.

\section{References}

1. Kitagawa S, Kitaura R, Noro S (2004) Functional Porous coordination polymers. Angewandte Chemie International Edition 43(18): 23342375 .

2. Batten R, Champness R, Chen M, Garcia Martinez, J Kitagawa, et al. (2013) Terminology of metal-organic frameworks and coordination polymers. Pure and Applied Chemistry (IUPAC) 85(8): 1715-1724.

3. Rowsell JLC, Yaghi OM (2004) Metal-organic frameworks: a new class of porous materials. Microporous Mesoporous Mater 73(1-2): 3-14.

4. Cheetham AK, Rao CNR, Feller RK (2006) Structural diversity and chemi- cal trends in hybrid inorganic-organic framework materials. Chem Commun 46: 4780-4795.

5. MO Keeffe (2009) Design of MOFs and intellectual content in reticular chemistry: A personal view. Chem Soc Rev 38(5): 1215-1217.

6. Long J, Yaghi O (2009) The pervasive chemistry of metal-organic frameworks. Editorial Chem Soc Rev 38(5): 1213-1214.

7. Sareeya B, Satoru J, Susumu K (2008) Chemistry and application of flexible coordination polymers. Science Technol Adv Mater 9(1).

8. Chen X, Ye B, Tong M (2005) Metal-organic molecular architectures with 2, 2-bipyridyl-like and carboxylate ligands. Coord chem Rev 249(5-6): 545-565.

9. Bernstein J, Paul M, Stephen G (2000) Morden Physics. Prentice-hall pp. 624.

10. Hung low F, Klausmeya K, Gary J (2009) Effect of anion and ligand ratio in self-assembled silver (I) complexes of 4-(diphenylpho sphinomethyl) pyridine and their derivatives with bipyridine ligands. Inorganic Chemica Acta 362(2): 426-436.

11. Ricci G, Sommazzi A, Masi F, Ricci M, Boglia A, et al. (2010) “Well-defined transition metal complexes with phosphorus and nitrogen ligands for 1,3-dienes polymerization". Coordination Chemistry Reviews 254(5-6): 661-676.

12. Wen M, Munakata M, Juenaga Y, Kurodasowa T, Maekawa M, et al. (2001) Silver (1) coordination polymers of cyclic sulphur ligand, 2,2,3-tetrahydro-4,4-dithia-1,1-binaphthylidene. Inorganica chimica Acta 322(1-2): 133-137.

13. Knaust J, Keller S (2002) A mixed-ligand coordination polymer from the in situ, $\mathrm{Cu}(\mathrm{I})$-medicated isomerization of Bis(4-pyridyl)ethylene. Inorganic Chemistry 41(22): 5650-5652.

14. Carlucci L, Ciani G, Proserpio O, Rizzato S (2002) New polymeric networks from silver (I) salts and the flexible ligands 1, 3-bis(4-pyridyl) propane (bpp): A systematic investigation of the effect of the counterions and a survey of the coordination polymers based on bpp. Cryst Eng Comm. 4(22): 12-16.

15. Ni L, Zhang R, Liu Q Xia W, Wang H, et al. (2009) pH and mole-ratio dependent formation of Zinc (II) coordination polymers with iminodiacetic acid synthesis, spectroscopic, crystalline structure and thermal studies. Journal of Solid State Chemistry 182(10): 2698.

16. Tong M, Hu S, Wang J, Kitagawa S, Ng S (2005) Supramolecular isomerism in cadmium hydroxide phases Temperature-Dependent synthesis and structure of photoluminescent coordination polymers $\alpha$ and $\beta$ $\mathrm{Cd}_{2}\left(\mathrm{OH}_{2}\right)(2,4-$ pyda). Crystal Growth and Design 5(3): 837-839.

17. Seetharaj R, Vandana PV, Arya P, S Mathew (2017) Dependence of solvents, $\mathrm{pH}$, molar ratio and temperature in tuning metal organic framework architecture. Arabian Journal of Chemistry.

18. Yaghi O, O Keeffe M (2005) Reticular Chemistry: Design, Synthesis, Properties and Applications of Metal-Organic Polyhedra and Frameworks. J Solid State Chemistry 178: 2409-2574.

19. Yang Y, Jiang G, Li YZ, Bai J, Pan Y, et al. (2006) "Synthesis, structures and properties of alkaline earth metal benzene-1,4-dioxylacetates with three-dimensional hybrid networks". Inorganica Chimica Acta 359 (10): 3257-3263.

20. Grubbs H, Luin S, Carraher C, Sheats J, Pittman C (1978) Organometallic Polymers, Academic Press, Inc, New York, pp. 129.

21. Pittman C, Becker E, Tsutsui M (1977) Organometallic Reactions. Plenum Publishing Corp. New York.

22. Foxman B, Janfmann J (1977) Polym Symposia. J Polym Sci 70: 31.

23. Tranchemontagne DJ, Hunt JR, Yaghi OM (2008) Room temperature syn- 
thesis of metal-organic frameworks: MOF-5, MOF-74, MOF- 177, MOF199, and IRMOF-0. Tetrahedron 64(36): 8553-8557.

24. Wang L, Zhang L, Song T, Li C, Xu J, et al. (2012) Solvothermal syntheses, structures and properties of two new In-MOFs based on rigid 1,4-naphthalenedicarboxylate ligand. Microporous and mesoporous materials 155: 281-286.

25. Yu Bin D, Ralph C, Mark D, Neil G, Uwe H, et al. (1999) Synthesis and characterization of one dimensional coordination Polymers generated from cadmium nitrate and Bipyridine ligands. Inorg Chem 38(13): 30563060 .

26. Krishnamurthy G, Agarwal S (2014) Room Temperature Synthesis and Characterization of a Zn (II) based Metal-organic Framework with Mixed Ligands, 1, 4- Benzenedicarboxylic Acid and 1-methyle Imidazole. Procedia Material science 5: 1258-1265.

27. Kitazawa T, Gomi T, Takahashi M, Takeda M, Enomoto $\mathrm{M}$, et al. (1996) Spin-crossover behavior of the coordination polymer $\left[\mathrm{Fe}^{11}\left(\mathrm{C}_{5} \mathrm{H}_{5} \mathrm{~N}\right)_{2} \mathrm{Ni}(\mathrm{CN})_{4}\right.$. J Mater Chem 6(1): 119-121.

28. Zhong Z, Seino H, Mizobe Y, Hidia M, Fujishima A, et al. (2000) A high spin cyanide-bridged Mn9W6 cluster( $S=39 / 2)$ with a full-capped $\mathrm{cu}-$ bane structure. J Am Chem Soc 122(12): 2952-2953.

29. Soma T, Iwamoto T (1996) Variation of multi-dimensional supramolecular structures built of the two-dimensional $[\mathrm{Cd}(\mathrm{py}) 2\{\mathrm{Ag}(\mathrm{CN}) 2\} 2] \mathrm{n}$ network: Three-dimensional textile structures of catena-poly[trans-bis(pyridine)cadmium (11)-di- $\mu$-\{dicyanorgentato (1)-N, N'\}]. J Incl Phen and Macrocyc Chem 26(1-3): 161-173.

30. Dursun K, Gunes S, Okan Z, Tunur H, Hakan D (2012) Synthesis of crystal structure of cyans-bridged three dimensional polyheteronuclear complex. Z Kristall org 227: 639-645.

31. Amish IS, Hemang MS, Purvesh JS, Dilipsinh SR (2017) Synthesis, Characterization and Antimicrobial Studies of Coordination Polymers. Journal of the Iranian Chemical Society 14: 1601-1612.

32. Sato 0, Tlyoda T, Fujishima A, Hashimoto K (1996) Electrochemically Tunable Magnetic Phase Transition in High-Tc Chromium Cyanide. Thin Film Science 271(5245): 49-51.

33. Jung OS, Kim YJ, Lee YA, Park JK, Chae Smart HK (2000) Molecular Helical Springs as Tunable Receptors. Journal of the American Chemical Society 122(41): 9921-9925.

34. Zhang L, Qin YY, Li ZJ, Lin QP, Cheng JK, et al. (2008) Topology Analysis and Nonlinear-Optical-Active Properties of Luminescent Metal-Organic Framework Materials Based on Zinc/Lead Isophthalates. Inorganic Chemistry 47(18): 8286-8598.

35. Katz MJ, Ramnial T, Yu HZ, Leznoff DB (2008) Polymorphism of $\mathrm{Zn}\left[\mathrm{Au}(\mathrm{CN})_{2}\right]_{2}$ and Its Luminescent Sensory Response to NH3 Vapor. Journal of the American Chemical Society 130(32): 10662-10673.

36. Jung OS, Kim YJ, Lee YA, Park JK, Chae Smart HK (2000) Molecular Helical Springs as Tunable Receptors. Journal of the American Chemical Society 122(41): 9921-9925.
37. Chin Ti C, Kenneth S (1993) One dimensional coordination polymers: Applications to materials science. Coordination Chemistry Reviews 128(1-2): 293-322.

38. Huang B, McGaughey A, Kaviany M (2007) Thermal conductivity of metal-organic framework 5 (MOF-5): Part I. Molecular dynamics simulations. International Journal of Heat and Mass Transfer 50(3-4): 393-404.

39. West A (1984) Solid State Chemistry and its Applications. Wiley, New York, pp. 582.

40. Bureekaew S, Shimomura S, Kitagawa S (2008) "Chemistry and application of flexible porous coordination polymers". Science and Technology of Advanced Materials 9(1): 014108.

41. Fromm K (2008) "Coordination polymer networks with s-block metal ions". Coord Chem Rev 252(8-9): 856-885.

42. Ellis D, Graham C (2007) Caged compounds: Photolease technology for control of cellular chemistry and physiology. Nature methods 4(8): 619628.

43. Horcajada P, Serre C, Maurin G, Ramsahye NA, Balas F, et al. (2008) Flexible Porous Metal-Organic Frameworks for a Controlled Drug Delivery. Journal of the American Chemical Society 130(21): 6774-6780.

44. An J, Geib SJ, Rosi NL (2009) Cation-Triggered Drug Release from a Porous Zinc-Adeninate Metal-Organic Framework. Journal of the American Chemical Society 131(24): 8376-8377.

45. Kesanli B, Cui Y, Smith M, Bittener E, Bockrath B, et al. (2005) Highly interpenetrated metalorganic frameworks for hydrogen storage. Angew Chem Int Edn 44(1): 72-75.

46. Kondo M, Yoshitomi T, Matsuzaka H, Kitagawa S, Seki K (1997) Three-Dimensional Framework with Channeling Cavities for Small Molecules: $\left\{\left[\mathrm{M}_{2}\left(4,4^{\prime}-\text {-bpy }\right)_{3}\left(\mathrm{NO}_{3}\right)_{4}\right] \cdot \mathrm{xH}_{2} \mathrm{O}\right\}_{\mathrm{n}}(\mathrm{M}-\mathrm{Co}, \mathrm{Ni}, \mathrm{Zn})^{+}$. Angew Chem Int Edn 36(16): 1725-1727.

47. Li J, Kuppler RJ, H Zhou H (2009) Selective gas adsorption and separation in metal-organic frameworks. Chem Soc Rev 38(5): 1477.

48. Duren T, Bae Y, Snurr RQ (2009) Using molecular simulation to characterize metal-organic frameworks for adsorption applications. Chem Soc Rev 38(5): 1237-1247.

49. Corma A, Garca H, Llabres X, Xamena I (2010) Engineering Metal Organic Frameworks for Heterogeneous Catalysis. Chem Rev 110(8): 46064655.

50. Lee J, Farha OK, Roberts J, Scheidt KA, Nguyen ST, et al. (2009) Metal-organic framework materials as catalysts. Chem Soc Rev 38(5): 1450.

51. Yang Y, Jiang G, Li YZ, Bai J, Pan Y, et al. (2006) "Synthesis, structures and properties of alkaline earth metal benzene-1,4-dioxylacetates with three-dimensional hybrid networks". Inorganica Chimica Acta 359 (10): 3257-3263.

52. Yu Bin D, Ralph C, Mark D, Neil G, Uwe H, et al. (1999) Synthesis and characterization of one dimensional coordination Polymers generated from cadmium nitrate and Bipyridine ligands. Inorg Chem 38(13): 30563060 . 
(c) (i) This work is licensed under Creative

Submission Link: Submit Article

DOI: 10.32474/AOICS.2018.01.000106

\section{AOICS}

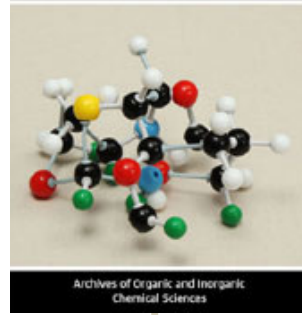

Archives of Organic and Inorganic Chemical Sciences

\section{Assets of Publishing with us}

- Global archiving of articles

- Immediate, unrestricted online access

- Rigorous Peer Review Process

- Authors Retain Copyrights

- Unique DOI for all articles 